\title{
Design of the Real-Time Simulation Experiment System of the Spread Spectrum System
}

\author{
Li Rong \\ College of Communication and Information Engineering, Xi'an \\ University of Science and Technology, Xi'an 710054, China \\ Cold3000@126.com
}

\begin{abstract}
Spread spectrum communication, a major course, has many features such as strong theory, abstract concept and rich relative techniques. This paper introduces the real-time simulation experiment system for spread spectrum communication based on MATLAB/Simulink, and realizes 4 experiments about the transmitters and receivers of direct sequence (DS). The Results show that this system have a good interactive interface, easy operation, high visualization and correct result. All of that are helpful to both the teaching of teacher and studying of students. It can improve the teaching effect and teaching quality.

Key words: spread spectrum communication; experiment system; MATLAB; Simulink

\section{Introduction}

Spread spectrum communication, being an important major course, has many features such as strong theory and rich relative techniques, which includes anti interference technology, modulation and demodulation technology and multiple access technology[1]. It is difficult for students to understand the abstract concepts. Furthermore, the students have few opportunities to design and realize the system because of the limited experimental conditions. Thus, the learning interest and the practical ability of students cannot be improved. The paper introduces the real time simulation system for spread spectrum communication based on MATLAB/Simulink. The system can be demonstrated in class to visualize the abstract concepts and the students will understand and master the knowledge more easily.
\end{abstract}

\section{Composition of the Experimental System}

In the paper, the direct sequence spread spectrum system is simulated. The system includes two modules: the transmitting terminal and receiving terminal. The experimental system covers the most knowledge of the spread spectrum 
communication course and can be demonstrated for the students in class. It can help the students to remember and comprehend the theory of the course.

\section{Design of the Experimental System}

\subsection{Module of the transmitting end}

The parameters of the module of the transmitting terminal are listed as follows: $1 \mathrm{~kb} / \mathrm{s}$ data rate and $127 \mathrm{~kb} / \mathrm{s}$ spread spectrum code rate. The $\boldsymbol{m}$ sequence is used as spread spectrum code, whose generating polynomial is $f(x)=1+x^{6}+x^{7}$ and cycle is 127 . The Bpsk modulation technology is utilized with $500 \mathrm{KHz}$ carrier frequency. The simulation block of the transmitting end is shown in Fig.1.

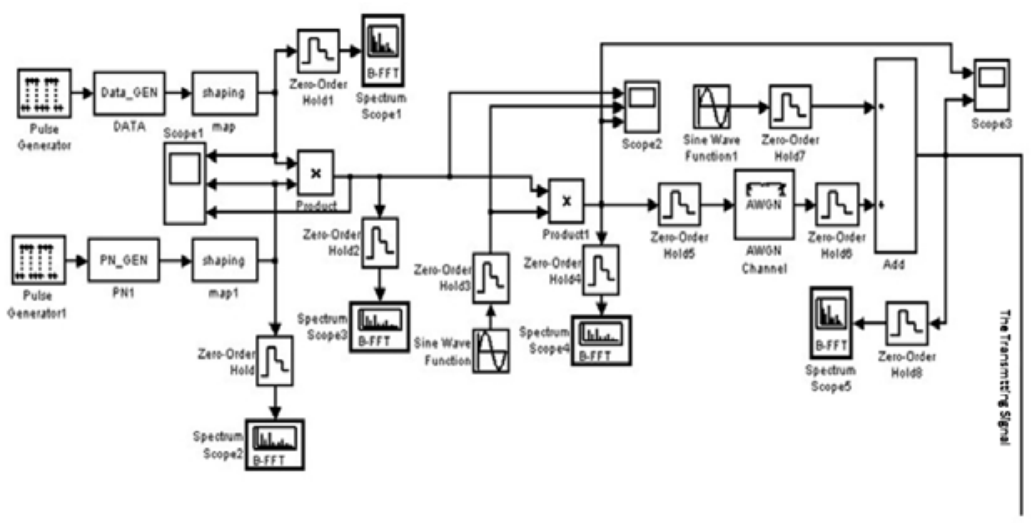

Fig.1: Simulation block of the transmitting end

The data of the transmitting terminal are produced by the Data_GEN module which applies the Function S Data_GEN to produce a random " 1 " or "0" sequence. The process is controlled by the time module Pulse_Generator. The spread spectrum code is produced by the PN_GEN module which applies the Function S PN_GEN to produce a $m$ sequence whose cycle is 127 . The process is controlled by clock module Pulse_Generator1. The negative logic mapping is accomplished by the shaping module. In the course of mapping the binary " 1 " is changed to " 1 " level and " 0 "is changed to " -1 " level. The spread spectrum modulation is completed by Product module. Besides, the radio frequency (RF) modulation is accomplished by Produce 1 module and the magnitude of sine carrier is 1 which is produced by Sine Wave Function module. The modulated RF signal passes through the additive white noise channel and the interference signal is the single frequency sine carrier produced by Sine Wave Function 1 module. In the course of simulation the signal to noise ratio is assumed to be $20 \mathrm{~dB}$. 


\subsection{Module of the receiving end}

Firstly, the frequency of the received signal is converted down to 250 $\mathrm{KHz}$ by heterodyne correlation dispreading device. Then the carrier is extracted and the data are regained by the Costas ring. The simulation block of the receiving end is shown in Fig. 2.

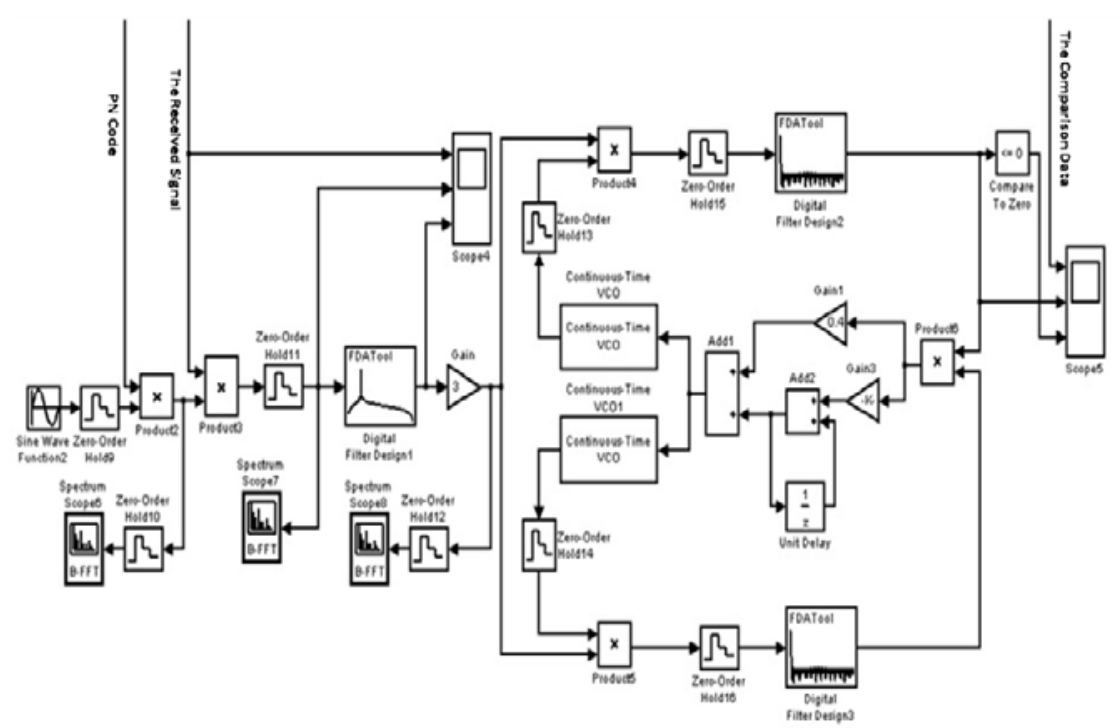

Fig.2: Simulation block of the receiving end

A local carrier with $250 \mathrm{KHz}$ frequency is produced by Sine Wave Function 2 module, which is modulated by the synchronized pseudo random code. Then the modulated signal is correlation processed with the received signal and the correlation processor is composed of Product3 and Digital Filter Design1 module. The Digital Filter Design1 module is a narrowband filter with $2 \mathrm{KHz}$ band whose center frequency is $250 \mathrm{KHz}$. The dispread signal is sent into the Costas ring to extract the medium frequency carrier and demodulate after amplified by the Gain module. The two-phase Costas ring is constructed because of Bpsk modulation technology. Besides, the static frequency of continuous time voltage controlled oscillator is $250 \mathrm{KHz}$, whose sensitivity of output frequency is $1 \mathrm{~Hz} / \mathrm{V}$. The filter is composed of Gain1, Gain3, Add2 and delay unit. Finally, the useful information is recovered after a simple judgment module.

\section{Realization of the experimental system}

\subsection{The experiment of the transmitting end}

The simulation results of spread spectrum and modulation are shown in Fig.3. 
Among these figures, (a) is the waveform after spread spectrum and (b) includes the waveforms before modulation and after modulation. (c) is the spectrum waveform of the baseband signal before spread spectrum and (d) shows the spectrum waveform of the signal after spread spectrum. (e) is the spectrum waveform after radio frequency modulation. The experimental results correspond to those of the theory.

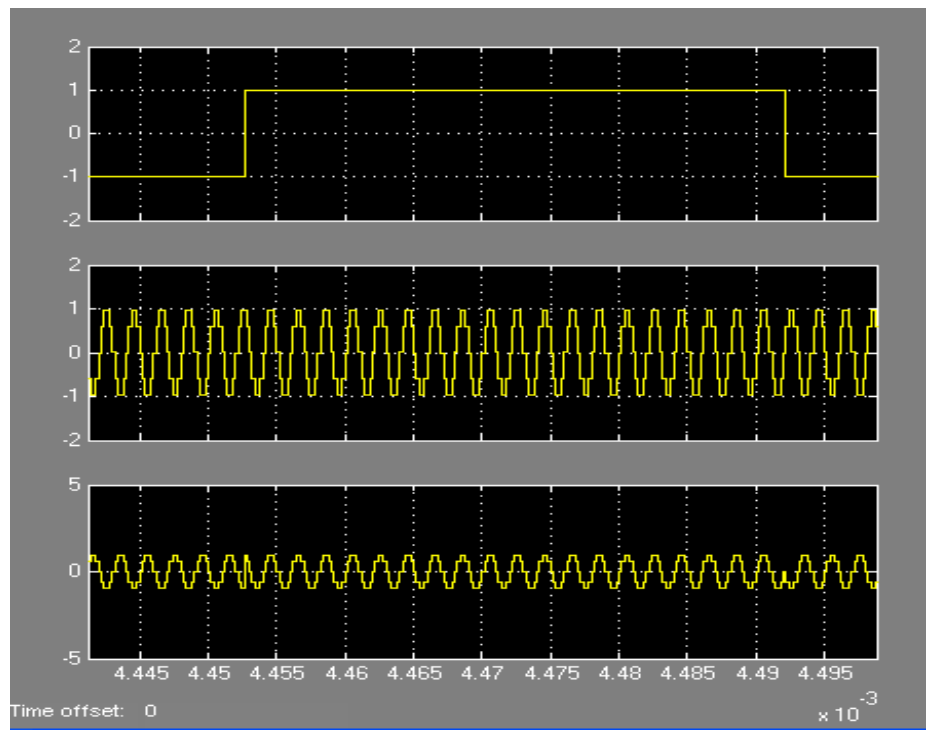

(a) waveforms of spread spectrum

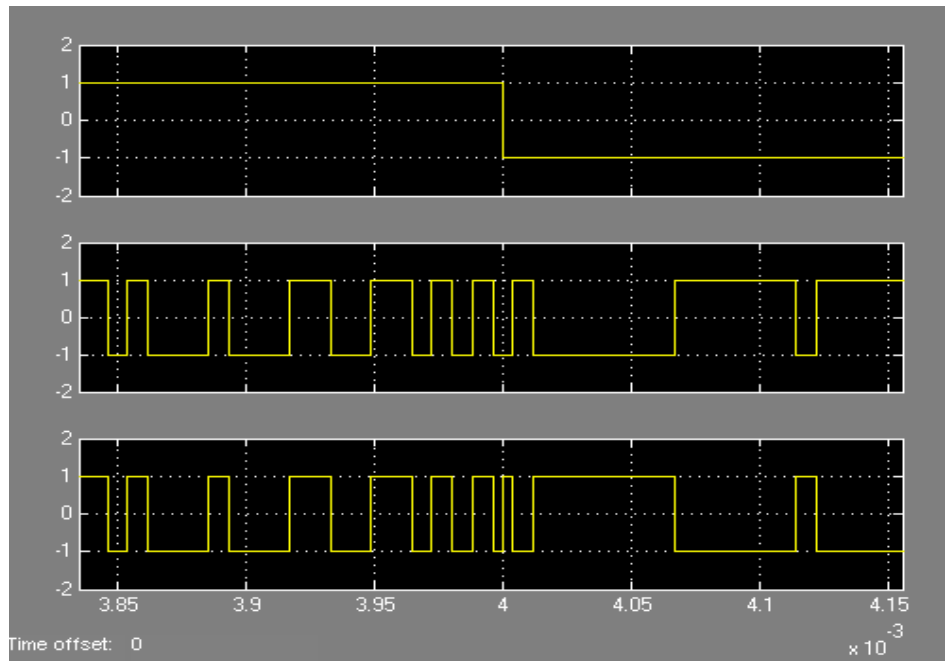

(b) waveforms of modulation 


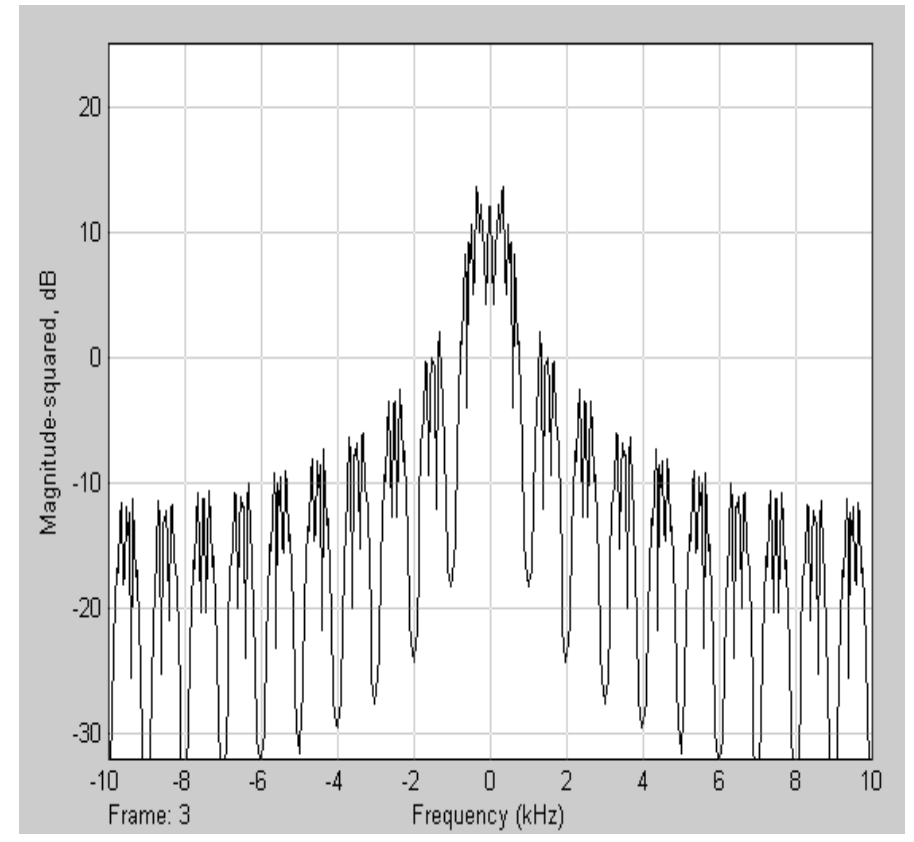

(c) spectrum waveform before spread spectrum

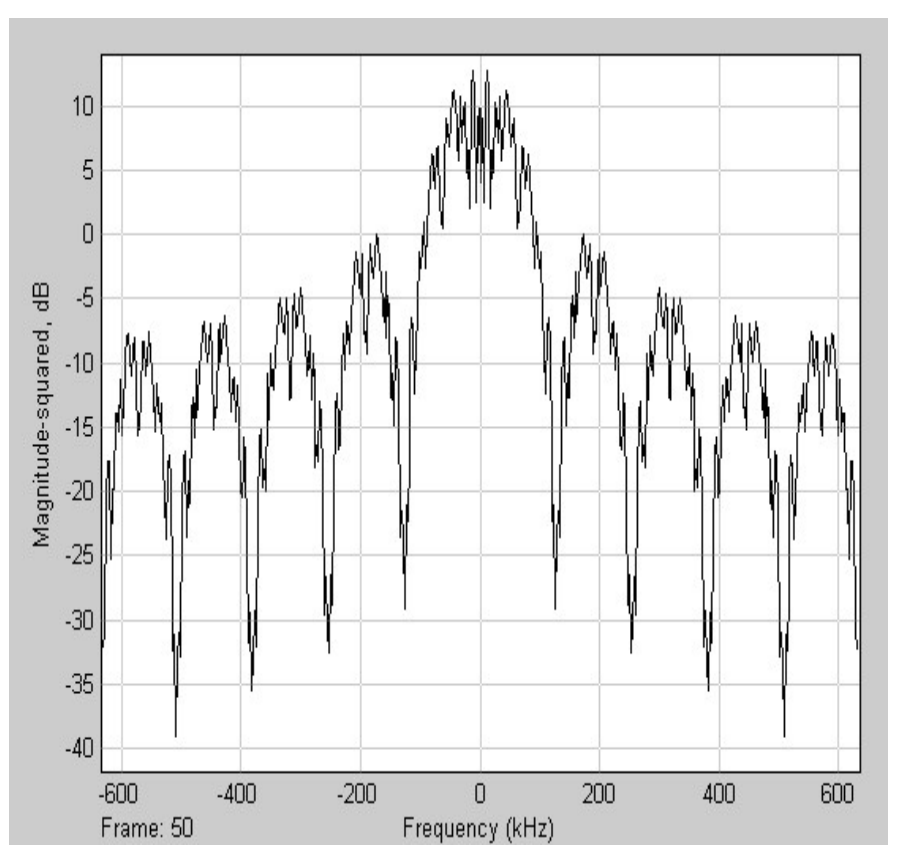

(d) spectrum waveform after spread spectrum 


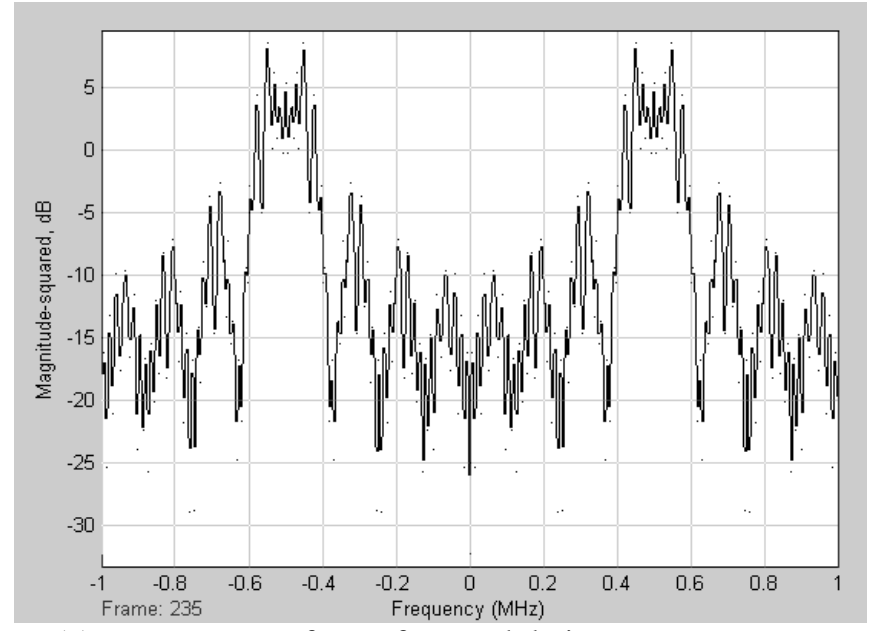

(e)spectrum waveform after modulation

Fig.3: the simulation results of spread spectrum and modulation

\subsection{The experiment of the receiving end}

The simulation waveforms of the receiving end are shown in Fig.4. The waveforms before and after correlation dispreading are illustrated in Figure (a). (b) and (c) include the spectrum waveforms before and after correlation dispreading. (d) is the comparison waveform between the demodulated signal and the original signal. All the experimental results are correct.

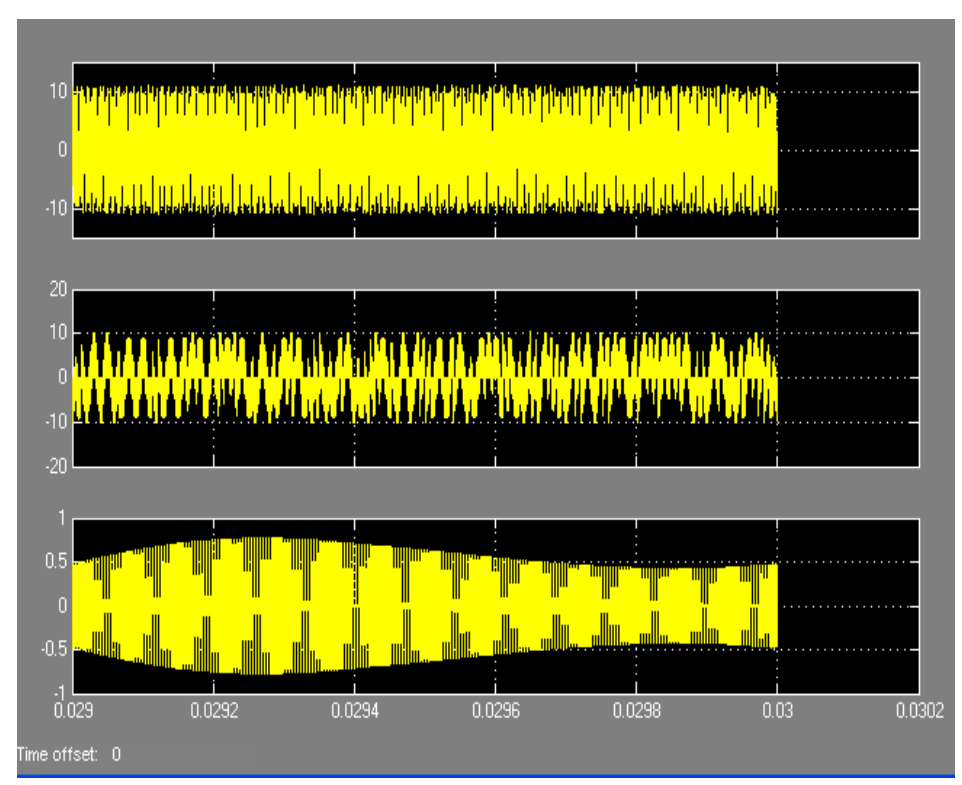

(a) waveforms of dispreading 


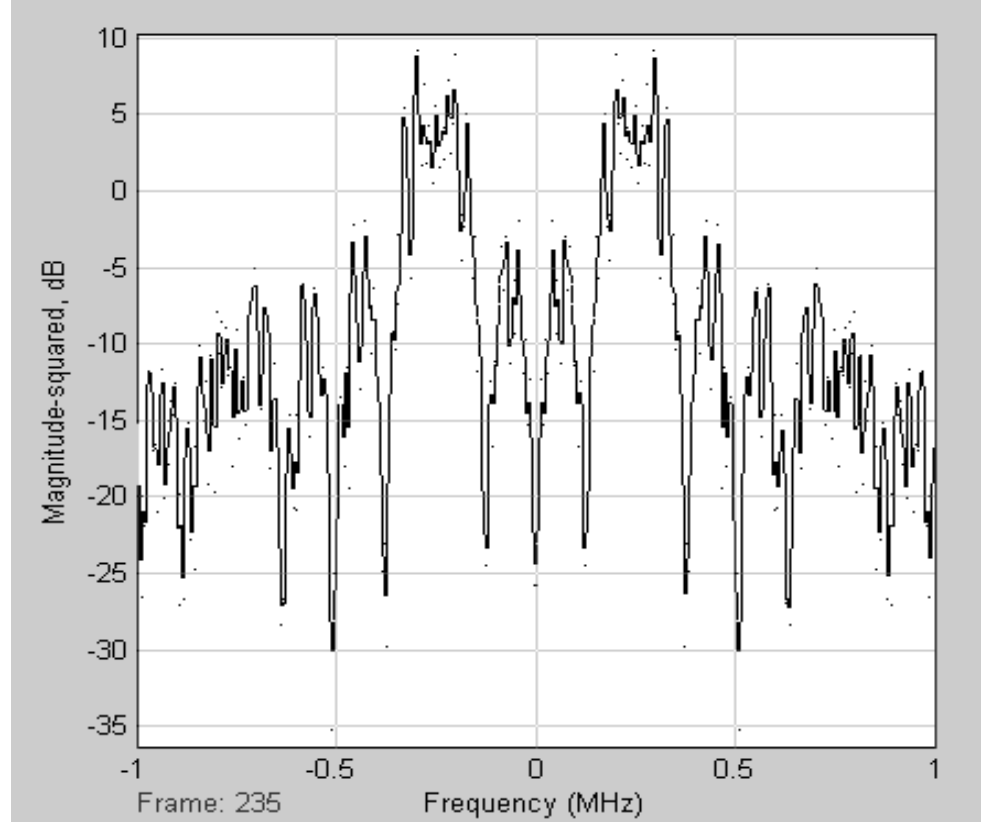

(b) spectrum waveform before Dispreading

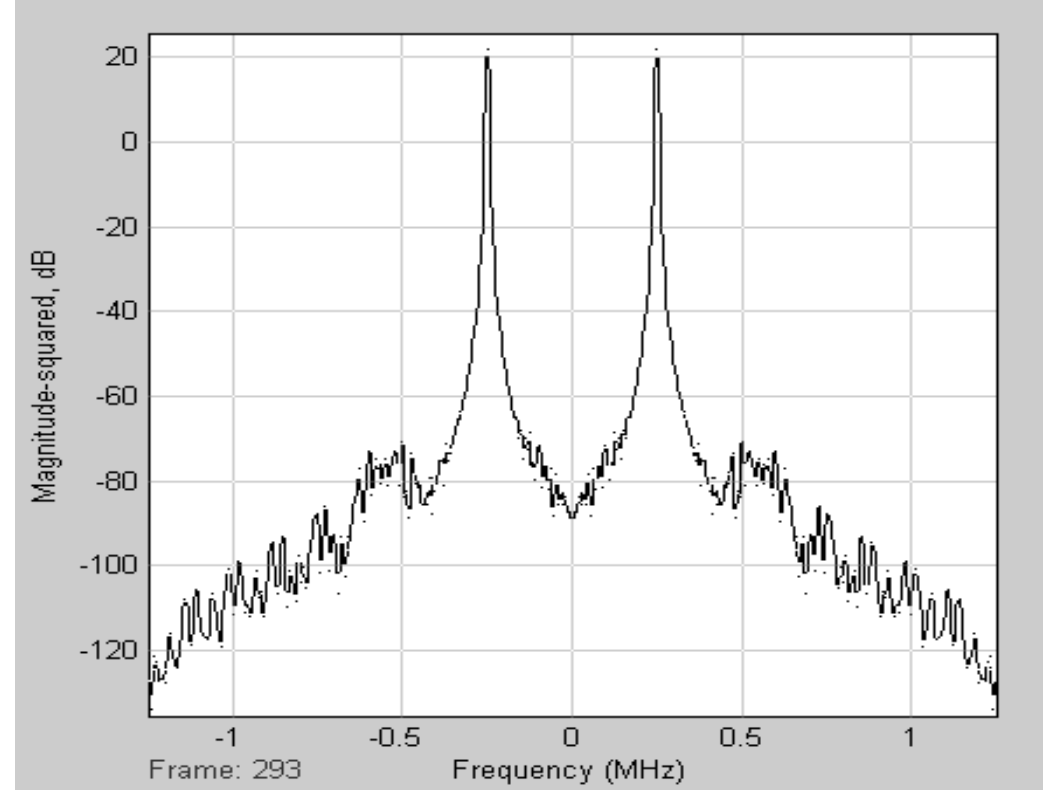

(c) spectrum waveform after dispreading 


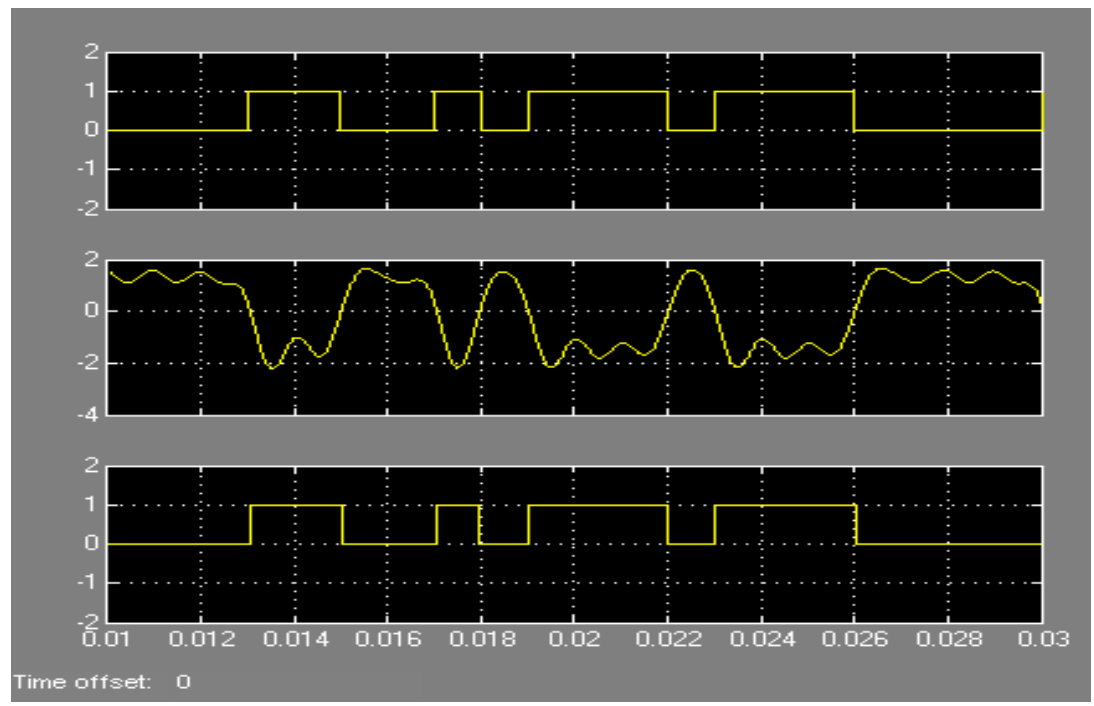

(d) waveforms of demodulation

Fig.4: the simulation results of dispreading and demodulation

\section{Conclusions}

The experimental results obtained by the real time simulation system are correct and the output values will be changed with the change of the input parameters. Therefore, the experimental system can be applied to the practical teaching to make the abstract theory become visualization. In a word, the simulation system can simplify the complexity of the teaching and provide some convenience for the students.

\section{References}

[1] Tian Ri-cai. Spread Spectrum Communication [M] (in Chinese). Tsinghua University Press, 2007.

[2] Wang Fang. Research and Practice of Flexible Experimental System for Communication Engineering Specialty based on Matlab Platform [J] (in Chinese). Experimental Technology and Management, 29(7), pp: 77-79, 2012.

[3] Zhao Guo-sheng. The Complete Learning Manual of Matlab [M] (in Chinese). Tsinghua University Press, 2014

[4] Ding Yi-nong. Simulink and Signal Processing [M] (in Chinese). Beihang University Press, 2014

[5] Bao Cheng-hong. Experimental Study of Communication Principles Based on Simulink [J] (in Chinese). Journal of Zhejiang Wanli University, 27(3), pp: 107-110, 2014. 